\title{
The incidence of gastrointestinal adverse events in patients with advanced non-small cell lung cancer (NSCLC) treated with PD-1 inhibitors: a meta-analysis
}

\author{
Geng-Wei Huo ${ }^{1,2 \#}$, Fu-Yi Zhu ${ }^{1 \#}$, Ran Zuo ${ }^{1 \#}$, Ying Song ${ }^{3}$, Wei-Dong Chen ${ }^{2}$, Wen-Ming Chen ${ }^{2}$, Hong-Mei \\ Zhang $^{2}$, Sha-Sha Jia ${ }^{2}$, Peng Chen ${ }^{1}$ \\ ${ }^{1}$ Department of Thoracic Oncology, Tianjin's Clinical Research Center for Cancer, Key Laboratory of Cancer Prevention and Therapy, National \\ Clinical Research Center for Cancer, Tianjin Medical University Cancer Institute and Hospital, Tianjin, China; ${ }^{2}$ Departmentof Oncology, Jining \\ No. 1 People's Hospital, Jining, China; ${ }^{3}$ Department of Pharmacy, Jining No. 1 People's Hospital, Jining, China \\ Contributions: (I) Conception and design: P Chen, GW Huo; (II) Administrative support: None; (III) Provision of study materials or patients: None; \\ (IV) Collection and assembly of data: FY Zhu, R Zuo, WD Chen, WM Chen; (V) Data analysis and interpretation: GW Huo, Y Song, HM Zhang, \\ SS Jia; (VI) Manuscript writing: All authors; (VII) Final approval of manuscript: All authors. \\ "These authors contributed equally to this work. \\ Correspondence to: Dr. Peng Chen. Department of Thoracic Oncology, Tianjin Medical University Cancer Institute and Hospital, North Huanhu \\ West Road, Hexi District, Tianjin 300060, China. Email: zhongliuke@foxmail.com.
}

Background: We conducted a meta-analysis to evaluates the incidence of the gastrointestinal (GI) adverse events with the use of PD-1 inhibitors among patients with advanced non-small cell lung cancer (NSCLC).

Methods: The PICOs (participants, intervention, comparison, and outcomes) elements were used for the selection of studies to meet the inclusion and exclusion criteria. Google Scholar, PubMed, Science Direct and proceedings of major oncology conferences were systematically searched from their inception to December 2020, to identify studies which reported the GI adverse events of PD-1 inhibitors among patients with NSCLC. Risks of bias were assessed by using a revised methodological index for nonrandomized studies (MINORS). Pooled incidences and weighted relative risk (RR) estimate for GI adverse events, the incidence of treatment discontinuation due to GI adverse events was also calculated. To perform the analysis of qualified studies, the model of random effects was used and the inconsistency of studies with the I2 index was investigated. OpenMeta 10.10, Stata 11.0 and RevMan 5.3 software were used for data analysis.

Results: The research included 15 studies comprising of a total of 3,716 patients. The incidences of allgrade GI symptoms were: diarrhea 8.6\% (95\% CI: 6.6-10.6\%), nausea 9.2\% (95\% CI: 7.3-11.0\%), vomiting $3.2 \%$ (95\% CI: $1.9-4.5 \%$ ), constipation $2.8 \%$ (95\% CI: $1.8-3.9 \%$ ), colitis $0.7 \%$ (95\% CI: $0.4-1.1 \%$ ), stomatitis (95\% CI: 1.0-2.7\%), and decreased appetite 10.0\% (95\% CI: 8.3-11.7\%). Therapy using PD-1 inhibitors was discontinued in $2.5 \%$ (95\% CI: $0.0-5.1 \%$ ) of patients with nausea, in $3.0 \%$ (95\% CI: $0.7-$ $5.3 \%$ ) of those with diarrhea, and in $45.7 \%$ (95\% CI: 20.6-70.7\%) of patients with colitis. Compared with chemotherapy, the use of PD-1 inhibitors showed significant increase in the occurrence of grade 1-4 colitis $(\mathrm{RR}=3.90,95 \% \mathrm{CI}: 1.41-10.81, \mathrm{P}=0.009)$ and grade 3-4 colitis ( $\mathrm{RR}=3.76,95 \% \mathrm{CI}: 1.07-13.26, \mathrm{P}=0.04)$.

Discussion: This meta-analysis provides a reliable estimate of the incidences of GI adverse events among NSCLC patients. Especially when colitis does occur, it often results in therapy discontinuation. Use of PD-1 inhibitors led to a higher incidence of colitis as compared to the use of chemotherapy.

Keywords: Non-small cell lung cancer (NSCLC); PD-1 inhibitors; gastrointestinal adverse events; meta-analysis

Submitted Jan 21, 2021. Accepted for publication May 20, 2021.

doi: $10.21037 /$ tcr-21-125

View this article at: https://dx.doi.org/10.21037/tcr-21-125 


\section{Introduction}

The GLOBOCAN study, conducted in 2018, reveals that lung cancer remains the leading cause of cancer-related mortality, causing an estimated 1.8 million deaths each year, globally (1). Depending on the histological features, lung cancers are classified as either small cell lung cancers (15$20 \%)$ or non-small cell lung cancers (NSCLCs) (80-85\%) (2).

Novel approaches to the treatment of NSCLC include immunotherapy targeting programmed cell death receptor- 1 and its ligand-1 and, hence, the use of PD-1/ PD-L1 inhibitors as well as cytotoxic T-lymphocyteassociated antigen 4 (CTLA-4). These innovative therapies have opened a new era in the treatment of lung cancer (3).

As immunotherapy continues to gain prominence in clinical practice, more and more adverse events are being observed. The adverse events associated with immunotherapy are different from those witnessed with the use of chemotherapy and small molecule targeted therapy. Reducing the incidence of adverse events is considered as important as is the prolongation of survival, especially for palliative treatment in some very late-stage cancer cases. Among adverse events, the incidence of gastrointestinal (GI) adverse events are second only to skin reactions among patients receiving immunotherapy.

GI adverse events may occur throughout the GI tract from upper involvement with oral aphthous ulcers, esophagitis, gastritis, to more distal involvement with enteritis and colitis (4). It is very important to further understand the incidence of GI adverse events in a larger scale of patients. This meta-analysis focuses on the incidence of GI adverse events with the use of PD-1 inhibitors and to find out if these adverse events were associated with treatment discontinuation. We present the following article in accordance with the PRISMA reporting checklist (available at https://dx.doi.org/10.21037/tcr-21-125).

\section{Methods}

\section{Inclusion and exclusion criteria}

The PICOs (participants, intervention, comparison, and outcomes) elements were used for selection of studies to meet the inclusion and exclusion criteria. Each letter represents a component: the patient population $(\mathrm{P})$, the interventions or exposure (I), the comparator group $(\mathrm{C})$, the outcome $(\mathrm{O})$, and the study design chosen $(\mathrm{S})$.
Duplicate articles were removed from retrieved studies before screening articles by title and abstract to identify studies that met the following inclusion criteria: (I) published articles in English and Chinese language, (II) investigated the GI adverse events of PD-1 inhibitor for the management of advanced/metastatic NSCLC patients, (III) reported the occurrence of GI adverse events. Articles with insufficient detail in the title and abstract to make a clear decision were included for further review. The earliest publication was selected for studies that have the same methodological descriptions and results. Full-text versions of all included publications were retrieved. Exclusion criteria are as follows: a study that reported (I) the outcomes of more than one PD-1 inhibitors without distinction, (II) received concurrent radiation or chemotherapy at the same time as an PD-1 inhibitors therapy, (III) pharmacokinetic or pharmacodynamic investigation, (IV) in vitro, molecular, or experimental investigations, or (V) qualitative information. We used not only the full text but also the appendix and the references of each article would be used as the information resources.

\section{Literature search}

Several electronic databases, including Google Scholar, PubMed, Cochrane library, Embase Science Direct and proceedings of major oncology conferences, were explored by using specific search terms and medical subject headings. Primarily, the search terms PD-1 inhibitors, NSCLC, GI adverse events were used followed by several other extensions including, but not limited to, the words nivolumab, pembrolizumab, diarrhea, nausea, vomiting, constipation, colitis, stomatitis and decreased appetite. The scope of the search encompassed research articles published before December 2020. In addition, bibliographies of important related papers were also screened.

\section{Quality assessment}

The quality of included studies was assessed independently by three authors (WM Chen, HM Zhang and SS Jia) using a revised methodological index for nonrandomized studies (MINORS). This tool consisted of 8 criteria for all studies, and quality score consisted of eight questions: a clearly stated aim, inclusion of consecutive patients, prospective collection of data, endpoints appropriate to the aim of the 
study, unbiased assessment of the study endpoint, follow-up period appropriate to the aim of the study, loss to follow up less than $5 \%$, prospective calculation of the study size. Each item is scored 0 (not reported), 1 (reported but inadequate) or 2 (reported and adequate) (5).

\section{Data collecting}

Studies and data extraction were conducted independently by two authors (Y Song and R Zuo) who reviewed and screened all studies for eligibility according to the inclusion criteria described previously. If there were any different opinions, the difference would be discussed with the third author (FY Zhu). Information was recorded regarding the baseline demographics, clinical, including oncology, profile, study design, methodology, and the number of patients who reported to have GI adverse events of grade 1-5, classified by version 4.0 of the Common Terminology Criteria for Adverse Events (CTCAE 4.0) (6).

\section{Statistical analyses}

We performed statistical analysis using the statistical software OpenMeta (version 10.10, opensource: Brown University, Providence, RI, USA) and Review Manager 5.3 (Cochrane Collaboration 2014, Nordic Cochrane Center, Copenhagen, Denmark). The prevalence of GI adverse events reported by the individual studies were pooled under the DerSimonian-Laird random-effects models (7). The relative risk (RR) and the corresponding $95 \%$ confidence intervals (CIs) were calculated in patients assigned to PD-1 inhibitors exclusively compared with those assigned to chemotherapy in the same trial. RR $>1.0$ indicates a higher risk or higher incidence of GI adverse events in patients treated with PD-1 inhibitors exclusively than those treated with chemotherapy. For the calculation of the RR, random or fixed-effect models were used, depending on the heterogeneity of included studies. The existence of heterogeneity was tested using the Chi-square statistics. The heterogeneity was quantified using the $\mathrm{I}^{2}$. For those variables with high heterogeneity $\left(\mathrm{P}<0.05\right.$ for $\mathrm{I}^{2}$ analysis), the data were analyzed using the random effect model. Otherwise, fixed-effect model was used for the data analysis (8). The results are presented in forest plots, together with pooled summary estimates and their corresponding $95 \%$ CI. Subgroup analysis was conducted according to different PD-1 inhibitors to explore the source of heterogeneity. The funnel plots were used to assessing the publication bias. The Egger linear regression test and Begg rank correlation methods were applied to determine publication bias were performed using Stata 11.0 software (Stata A Corp., College Station, Texas, USA). A two-tailed $\mathrm{P}$ value of less than 0.05 was considered statistically significant.

\section{Results}

\section{Literature search results}

Using the research search strategy, a total of 1,426 potentially relevant records were identified from databases and conferences. The selection process and reasons for exclusion of ineligible studies are presented in Figure 1. Some 1,411 studies were excluded after screening the abstract and full text. Accordingly, a total of 15 studies were considered eligible for the meta-analysis. These were clinical trials published between 2015 and 2019 with the following distribution: six phase III trials, one phase II/III trial, four phase II trials and four phase I trials (9-23).

\section{Characteristics of the selected studies}

The socio-demographic and clinical profile of the patients in the studies included in the meta-analysis are presented in Table 1. A total of 3,716 patients with advanced unresectable recurrent or metastatic NSCLC were enrolled. Out of these, 1,693 patients received nivolumab whereas 2,023 patients were on treatment involving pembrolizumab. All of the PD-1 inhibitors were administered as monotherapy. The age range of the patients was 27-93 years and comprised of fewer females $(34.8 \%)$. The minority of patients $(18.8 \%)$ had never smoked before. All of the studies used the National Cancer Institute CTCAE, version 4.0 to define the grade of toxicity.

The MINORS was used to evaluate the quality of each study. As shown in Table 1, The quality of included studies was high. Treatment-related GI adverse events occurring in patients with advanced NSCLC treated with anti-PD-1 therapy are presented in Table 2.

\section{Incidence of diarrhea associated with the use of PD-1 inbibitor}

All the 15 studies evaluated the incidence of diarrhea associated with the use of PD-1 inhibitor in 3,716 patients 


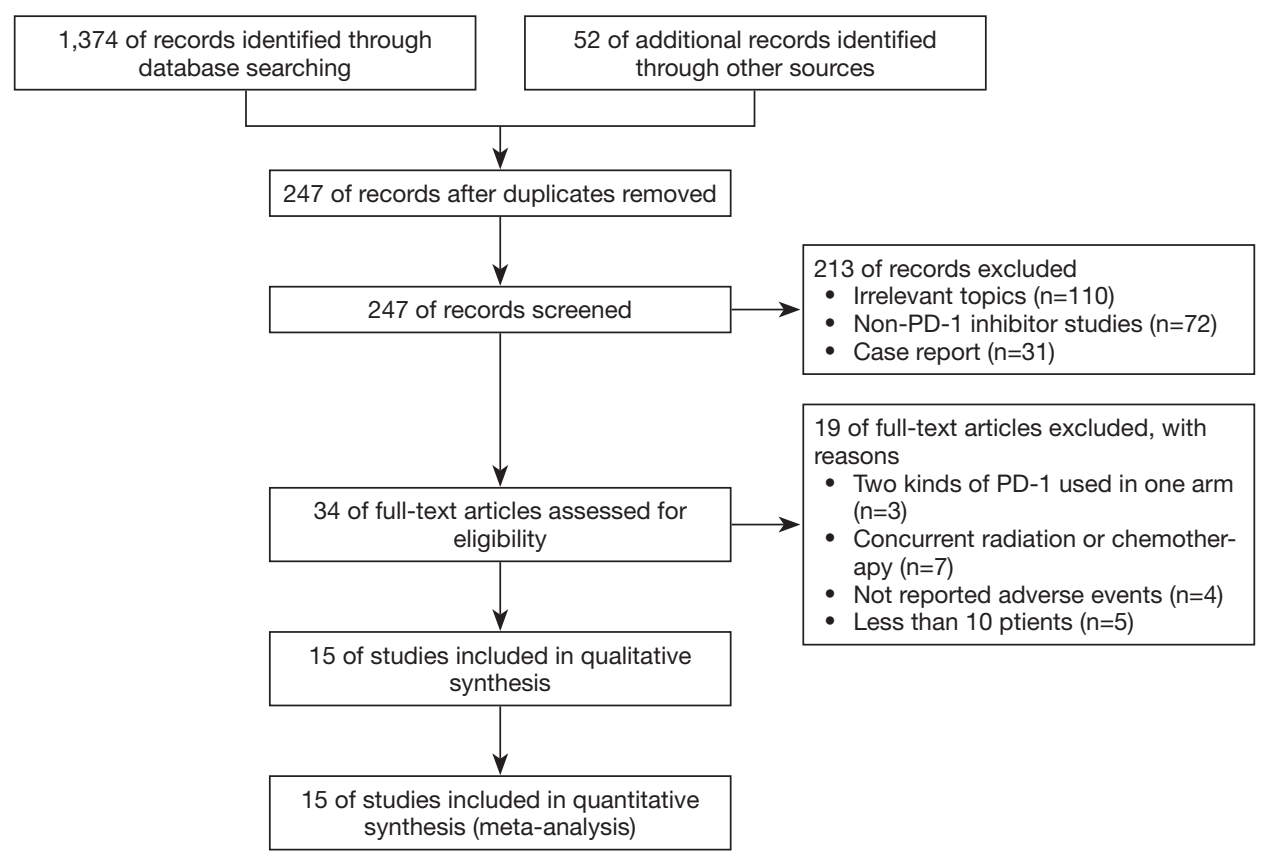

Figure 1 Flow diagram of included studies.

with advanced/metastatic NSCLC (Table 3). The incidence of all-grade [1-4] diarrhea associated with PD-1 inhibitors was $8.6 \%$ (95\% CI: 6.6-10.6\%). When stratified by severity of diarrhea, the incidence was $7.6 \%$ (95\% CI: $5.8-9.4 \%$ ) for grade $1-2$ diarrhea and $0.5 \%$ (95\% CI: $0.3-0.8 \%)$ for grade 3-4 diarrhea. Notably, the occurrence of diarrhea was similar in studies using either nivolumab or pembrolizumab, with incidences of $8.6 \%$ (95\% CI: $5.3-12.0 \%$ ) and $8.1 \%$ (95\% CI: $5.7-10.5 \%$ ), respectively.

\section{Incidence of nausea associated with PD-1 inhibitor}

Thirteen studies $(9-13,15-22)$ evaluated the incidence of nausea associated with the use of PD-1 inhibitor in 3,361 patients with advanced/metastatic NSCLC (Table 4). The incidence of all-grade [1-4] nausea associated with PD-1 inhibitors was $9.2 \%$ (95\% CI: $7.3-11.0 \%$ ), $8.8 \%$ (95\% CI: $7.0-10.6 \%)$ for grade $1-2$ nausea and $0.2 \%(95 \%$ CI: $0.1-0.4 \%)$ for grade 3-4 nausea. Using nivolumab or pembrolizumab, with incidences of $10.4 \%$ (95\% CI: $7.5-$ $13.3 \%$ ) and $7.9 \%$ (95\% CI: 5.4-10.4\%), respectively.

\section{Incidence of vomiting associated with PD-1 inbibitor}

Eleven studies (9-13,15,18,19,21-23) evaluated the incidence of vomiting associated with the use of PD-1 inhibitor in 2,943 patients with advanced/metastatic NSCLC (Table 5). The incidence of all-grade [1-4] vomiting was 3.2\% (95\% CI: $1.9-4.5 \%$ ). $3.0 \%$ (95\% CI: $1.7-4.4 \%$ ) for grade $1-2$ vomiting and $0.2 \%$ (95\% CI: $0.0-0.3 \%$ ) for grade $3-4$ vomiting. Using nivolumab or pembrolizumab, with incidences of $3.8 \%$ (95\% CI: $1.8-5.8 \%$ ) and $2.6 \%$ (95\% CI: $1.7-3.4 \%$ ), respectively.

\section{Incidence of constipation associated with PD-1 inbibitor}

Eleven studies (1,9-13,15,17-19,21,22) evaluated the incidence of constipation associated with the use of PD-1 inhibitor in 2,641 patients with advanced/metastatic NSCLC (Table 6). The incidence of all-grade [1-4] constipation was $2.8 \%$ (95\% CI: $1.8-3.9 \%$ ), $2.7 \%$ (95\% CI: $1.7-3.8 \%)$ for grade $1-2$ constipation and $0.2 \%(95 \%$ CI: $0.0-0.3 \%$ ) for grade 3-4 constipation. Using nivolumab or pembrolizumab, with incidences of $3.7 \%$ (95\% CI: 2.0 $5.4 \%$ ) and $1.8 \%$ (95\% CI: $0.8-2.8 \%$ ), respectively.

\section{Incidence of colitis associated with PD-1 inbibitor}

Nine studies $(9,10,14,16,18-21,23)$ evaluated the incidence of colitis associated with the use of PD-1 inhibitor in 2,359 patients with advanced/metastatic NSCLC (Table 7). 


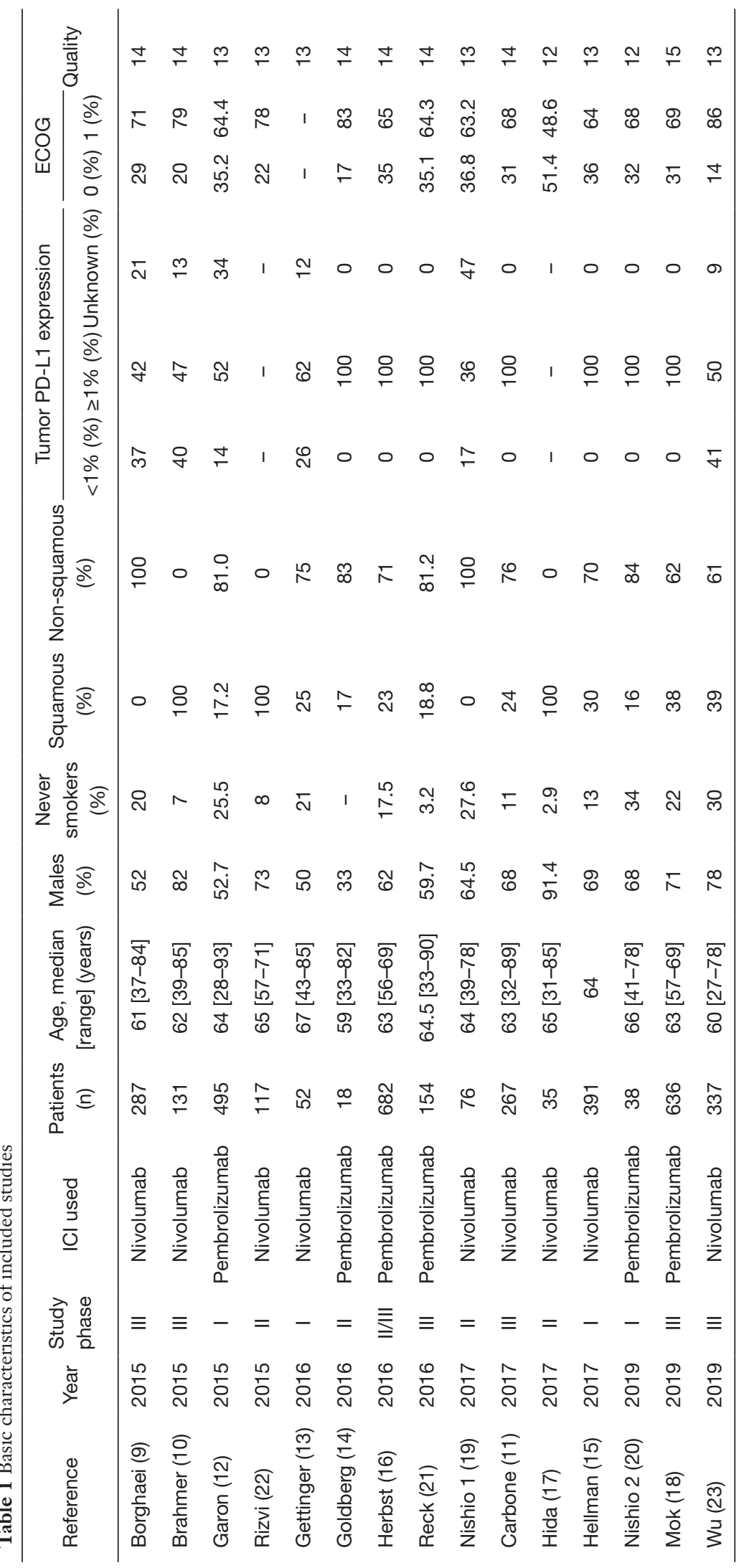




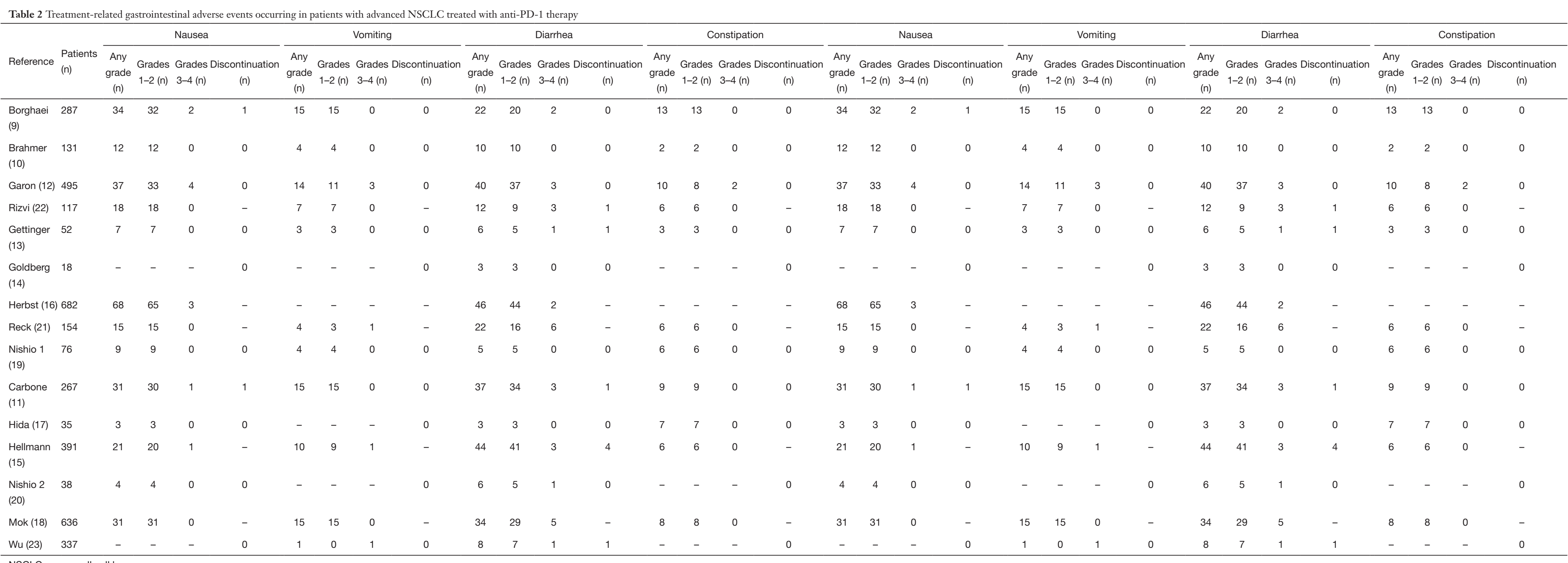


Table 3 Incidence of diarrhea with anti-PD-1 therapy

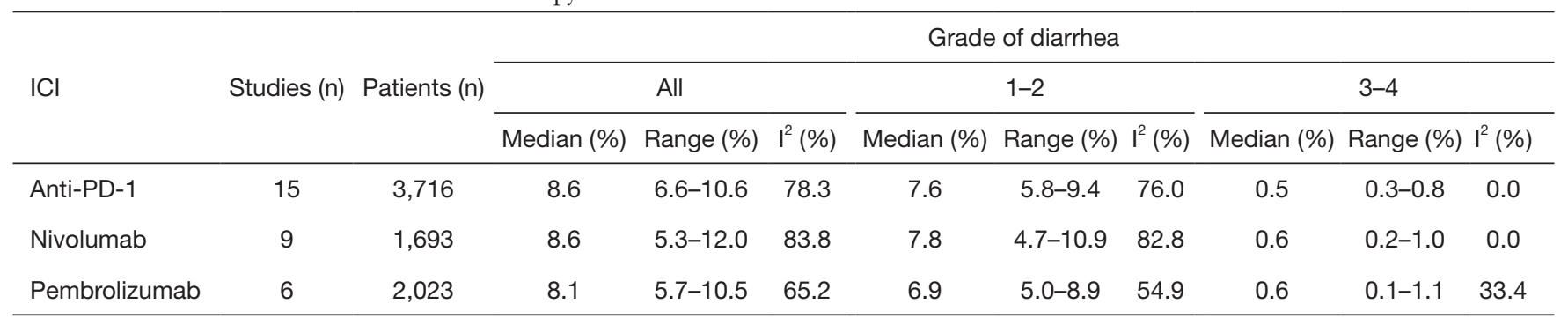

$\mathrm{ICl}$, immune checkpoint inhibitor.

Table 4 Incidence of nausea with anti-PD-1 therapy

\begin{tabular}{|c|c|c|c|c|c|c|c|c|c|c|c|}
\hline \multirow{2}{*}{$\mathrm{ICl}$} & \multirow{2}{*}{ Studies (n) } & \multirow{2}{*}{ Patients (n) } & \multicolumn{9}{|c|}{ Grade of nausea } \\
\hline & & & Median (\%) & Range (\%) & $\mathrm{I}^{2}(\%)$ & Median (\%) & Range (\%) & $\mathrm{I}^{2}(\%)$ & Median (\%) & Range (\%) & $\mathrm{I}^{2}(\%)$ \\
\hline Anti-PD-1 & 13 & 3,361 & 9.2 & $7.3-11.0$ & 67.8 & 8.8 & $7.0-10.6$ & 66.4 & 0.2 & $0.1-0.4$ & 0.0 \\
\hline Nivolumab & 8 & 1,356 & 10.4 & $7.5-13.3$ & 63.6 & 10.2 & $7.3-13.1$ & 64.1 & 0.4 & $0.4-0.7$ & 0.0 \\
\hline
\end{tabular}

$\mathrm{ICI}$, immune checkpoint inhibitor.

Table 5 Incidence of vomiting with anti-PD-1 therapy

\begin{tabular}{|c|c|c|c|c|c|c|c|c|c|c|c|}
\hline \multirow{2}{*}{$\mathrm{ICl}$} & \multirow{2}{*}{ Studies (n) } & \multirow{2}{*}{ Patients (n) } & \multicolumn{9}{|c|}{ Grade of vomiting } \\
\hline & & & Median (\%) & Range (\%) & $\mathrm{I}^{2}(\%)$ & Median (\%) & Range (\%) & $I^{2}(\%)$ & Median (\%) & Range (\%) & $\mathrm{I}^{2}(\%)$ \\
\hline Anti-PD-1 & 11 & 2,943 & 3.2 & $1.9-4.5$ & 80.0 & 3.0 & $1.7-4.4$ & 83.8 & 0.2 & $0.0-0.3$ & 0.0 \\
\hline Nivolumab & 8 & 1,658 & 3.8 & $1.8-5.8$ & 83.4 & 3.7 & $1.7-5.8$ & 85.8 & 0.3 & $0.0-0.5$ & 0.0 \\
\hline
\end{tabular}

$\mathrm{ICI}$, immune checkpoint inhibitor.

Table 6 Incidence of constipation with anti-PD-1 therapy

\begin{tabular}{|c|c|c|c|c|c|c|c|c|c|c|c|}
\hline \multirow{3}{*}{$\mathrm{ICl}$} & \multirow{3}{*}{ Studies (n) } & \multirow{3}{*}{ Patients (n) } & \multicolumn{9}{|c|}{ Grade of constipation } \\
\hline & & & \multicolumn{3}{|c|}{ All } & \multicolumn{3}{|c|}{$1-2$} & \multicolumn{3}{|c|}{$3-4$} \\
\hline & & & Median (\%) & Range (\%) & $\mathrm{I}^{2}(\%)$ & Median (\%) & Range (\%) & $\mathrm{I}^{2}(\%)$ & Median (\%) & Range (\%) & $I^{2}(\%)$ \\
\hline Anti-PD-1 & 11 & 2,641 & 2.8 & $1.8-3.9$ & 60.4 & 2.7 & $1.7-3.8$ & 61.1 & 0.2 & $0.0-0.3$ & 0.0 \\
\hline Nivolumab & 8 & 1,356 & 3.7 & $2.0-5.4$ & 63.1 & 3.7 & $2.0-5.4$ & 63.1 & 0.2 & $0.0-0.4$ & 0.0 \\
\hline
\end{tabular}

$\mathrm{ICI}$, immune checkpoint inhibitor. 
Table 7 Incidence of colitis with anti-PD-1 therapy

\begin{tabular}{|c|c|c|c|c|c|c|c|c|c|c|c|}
\hline \multirow{3}{*}{$\mathrm{ICl}$} & \multirow{3}{*}{ Studies (n) } & \multirow{3}{*}{ Patients (n) } & \multicolumn{9}{|c|}{ Grade of colitis } \\
\hline & & & \multicolumn{3}{|c|}{ All } & \multicolumn{3}{|c|}{$1-2$} & \multicolumn{3}{|c|}{$3-4$} \\
\hline & & & Median (\%) & Range (\%) & $\mathrm{I}^{2}(\%)$ & Median (\%) & Range (\%) & $\mathrm{I}^{2}(\%)$ & Median (\%) & Range (\%) & $\mathrm{I}^{2}(\%)$ \\
\hline Anti-PD-1 & 9 & 2,359 & 0.7 & $0.4-1.1$ & 0.0 & 0.4 & $0.1-0.6$ & 0.0 & 0.4 & $0.2-0.7$ & 0.0 \\
\hline Nivolumab & 4 & 831 & 0.3 & $0.0-0.6$ & 0.0 & 0.3 & $0.0-0.7$ & 0.0 & 0.4 & $0.0-0.8$ & 0.0 \\
\hline
\end{tabular}

$\mathrm{ICl}$, immune checkpoint inhibitor.

Table 8 Incidence of stomatitis with anti-PD-1 therapy

\begin{tabular}{|c|c|c|c|c|c|c|c|c|c|c|c|}
\hline \multirow{2}{*}{$\mathrm{ICl}$} & \multirow{2}{*}{ Studies (n) } & \multirow{2}{*}{ Patients (n) } & \multicolumn{9}{|c|}{ Grade of stomatitis } \\
\hline & & & Median (\%) & Range (\%) & $I^{2}(\%)$ & Median (\%) & Range (\%) & $I^{2}(\%)$ & Median (\%) & Range (\%) & $\mathrm{I}^{2}(\%)$ \\
\hline Anti-PD-1 & 6 & 2,102 & 1.8 & $1.0-2.7$ & 46.3 & 1.8 & $1.0-2.6$ & 41.9 & 1.0 & $0.0-0.3$ & 0.0 \\
\hline Nivolumab & 3 & 630 & 1.6 & $0.3-2.9$ & 31.7 & 1.6 & $0.3-2.9$ & 31.7 & 0.2 & $0.0-0.5$ & 0.0 \\
\hline
\end{tabular}

$\mathrm{ICl}$, immune checkpoint inhibitor.

The incidence of all-grade [1-4] colitis was $0.7 \%$ (95\% CI: $0.4-1.1 \%$ ), $0.4 \%$ (95\% CI: $0.1-0.6 \%$ ) for grade $1-2$ colitis and $0.4 \%$ (95\% CI: $0.2-0.7 \%$ ) for grade $3-4$ colitis. Using nivolumab or pembrolizumab, with incidences of $0.3 \%(95 \%$ CI: $0.0-0.6 \%$ ) and $1.1 \%$ (95\% CI: $0.5-1.6 \%)$, respectively.

\section{Incidence of stomatitis associated with PD-1 inbibitor}

Six studies $(9,11,16,18,19,21)$ evaluated the incidence of stomatitis associated with the use of PD-1 inhibitor in 2,102 patients with advanced/metastatic NSCLC (Table 8). The incidence of all-grade [1-4] stomatitis was $1.8 \%$ (95\% CI: $1.0-2.7 \%$ ), $1.8 \%$ (95\% CI: $1.0-2.6 \%$ ) for grade 1-2 stomatitis and $1.0 \%$ (95\% CI: $0.0-0.3 \%$ ) for grade 3-4 stomatitis. Using nivolumab or pembrolizumab, with incidences of $1.6 \%$ (95\% CI: $0.3-2.9 \%$ ) and $2.1 \%$ (95\% CI: $0.7-3.5 \%)$, respectively.

\section{Incidence of decreased appetite associated with PD-1 inbibitor}

Fourteen studies (9-12,14-23) evaluated the incidence of decreased appetite associated with the use of PD-1 inhibitor in 3,664 patients with advanced/metastatic NSCLC (Table 9). The incidence of all-grade [1-4] decreased appetite was $10.0 \%$ (95\% CI: $8.3-11.7 \%), 9.5 \%$ (95\% CI: 7.8-11.2\%) for grade 1-2 decreased appetite and 0.3\% (95\% CI: $0.1-$ $0.5 \%$ ) for grade 3-4 decreased appetite. Using nivolumab or pembrolizumab, with incidences of $10.5 \%$ (95\% CI: 8.0 $13.1 \%$ ) and $9.5 \%$ (95\% CI: 7.0-12.1\%), respectively.

\section{Incidence of treatment-related death and therapy discontinuation}

No treatment-related deaths attributable to GI adverse events occurred with the use of PD-1 inhibitors. Discontinuation of treatment was evaluated in 8 studies $(9-13,17,19,20)$ due to all-grade nausea. It was responsible for treatment discontinuation in $2.5 \%$ (95\% CI: $0.0-5.1 \%$ ) patients who developed nausea, whereas nausea led to termination of therapy in $0.14 \%$ of the patients on treatment using PD-1 inhibitors.

In 12 studies (9-15,17,19,20,22,23) comprising 2,244 patients, diarrhea was cited as the cause of treatment discontinuation in $3.0 \%$ (95\% CI: 0.7-5.3\%) patients who developed diarrhea. There was an overall discontinuation rate of $0.22 \%$ due to diarrhea among patients who received PD-1 inhibitors.

Among 887 patients $(9,10,14,19,20,23)$ available for evaluation, 7 of them developed colitis. Out of these, three patients (45.7\% , 95\% CI: 20.6-70.7\%) discontinued therapy. 
Table 9 Incidence of decreased appetite with anti-PD-1 therapy

\begin{tabular}{|c|c|c|c|c|c|c|c|c|c|c|c|}
\hline \multirow{2}{*}{$\mathrm{ICl}$} & \multirow{2}{*}{ Studies (n) } & \multirow{2}{*}{ Patients (n) } & \multicolumn{9}{|c|}{ Grade of decreased appetite } \\
\hline & & & Median (\%) & Range (\%) & $\mathrm{I}^{2}(\%)$ & Median (\%) & Range (\%) & $\mathrm{I}^{2}(\%)$ & Median (\%) & Range (\%) & $I^{2}(\%)$ \\
\hline Anti-PD-1 & 14 & 3,664 & 10.0 & $8.3-11.7$ & 60.8 & 9.5 & $7.8-11.2$ & 62.0 & 0.3 & $0.1-0.5$ & 0.0 \\
\hline Nivolumab & 8 & 1,641 & 10.5 & $8.0-13.1$ & 63.1 & 10.2 & $7.8-12.7$ & 60.1 & 0.2 & $0.0-0.4$ & 0.0 \\
\hline
\end{tabular}

$\mathrm{ICl}$, immune checkpoint inhibitor.

Table 10 Basic characteristics and the occurrence of colitis in the selected study of anti PD-1 monotherapy versus chemotherapy

\begin{tabular}{|c|c|c|c|c|c|c|c|c|c|}
\hline Reference & Experimental arm & Control arm & $\begin{array}{l}\text { Experimental arm } \\
\text { patients/control } \\
\text { arm patients }(n)\end{array}$ & \multicolumn{3}{|c|}{ Anti-PD-1 } & \multicolumn{3}{|c|}{ Chemotherapy } \\
\hline Brahmer (10) & Nivolumab & Docetaxel & $131 / 129$ & 1 & 0 & 1 & 0 & 0 & 0 \\
\hline Mok (18) & Pembrolizumab & $\begin{array}{l}\text { Investigator's choice of } \\
\text { carboplatin plus paclitaxel or } \\
\text { pemetrexed chemotherapy }\end{array}$ & $636 / 615$ & 7 & 3 & 4 & 2 & 1 & 1 \\
\hline
\end{tabular}

Overall, discontinuation of treatment because of colitis occurred in $0.30 \%$ of all patients who received PD- 1 inhibitors.

None of the patients treated with PD-1 inhibitors discontinued therapy because of vomiting, constipation, pancreatitis, stomatitis and decreased appetite.

\section{Comparison of the incidence of colitis between PD-1 inhibitors and chemotherapy}

Six studies reported the incidence of colitis between PD-1 inhibitors monotherapy and chemotherapy in patients with advanced/metastatic NSCLC. Collectively, there were 2,227 patients who were treated with PD-1 inhibitors monotherapy and 1,627 who were managed using chemotherapy $(9,10,16,18,21,23)$ (Table 10).

The pooled results of the meta-analysis are presented in Figure 2. The heterogeneity test indicated that a fixed-effects model could be selected. Compared with chemotherapy, the use of a PD-1 inhibitor showed significant increase in the occurrence of grade 1-4 and grade 3-4 colitis, with pooled RR of 3.90 (95\% CI: 1.41-10.81, $\mathrm{P}=0.009)$ and 3.76 (95\% CI: $1.07-13.26, \mathrm{P}=0.04)$, respectively. However, there was no significant increase in grade $1-2$ colitis $(\mathrm{RR}=2.46,95 \%$ CI: $0.68-8.88, \mathrm{P}=0.17$ ).

As shown in Tables 11,12, the sensitivity analysis was performed to detect whether the results could have an impact on the PD-1 inhibitors (grade $1-4$, grade $3-4$ colitis), respectively.

In addition, the funnel plots for the RR of grade 1-4 and grade 3-4 colitis showed that each of the studies was arranged symmetrically on either side of the funnel (Figures 3,4). The Egger linear regression test and Begg rank correlation method verified that there was no obvious publication bias (Egger's test: $t=0.12, \mathrm{P}=0.909$; Begg' test: $z=0.75, \mathrm{P}=0.806$ ). Collectively, there was no significant publication bias in our meta-analysis. 


\begin{tabular}{|c|c|c|c|c|c|c|c|c|c|}
\hline \multirow[b]{2}{*}{ Study or Subgroup } & \multicolumn{2}{|c|}{ Anti-PD-1 therapy } & \multicolumn{2}{|c|}{ Chemotherapy } & \multirow[b]{2}{*}{ Weight } & \multirow{2}{*}{$\begin{array}{c}\text { Risk Ratio } \\
\text { M-H. Fixed, } 95 \% \mathrm{Cl}\end{array}$} & \multirow{2}{*}{\multicolumn{2}{|c|}{$\begin{array}{c}\text { Risk Ratio } \\
\text { M-H, Fixed, } 95 \% \mathrm{Cl}\end{array}$}} & \\
\hline & Events & Total & Events & Total & & & & & \\
\hline Borghaei 2015 (9) & 2 & 287 & 0 & 268 & $10.5 \%$ & $4.67[0.23,96.83]$ & & & \\
\hline Brahmer 2015 (10) & 1 & 131 & 0 & 129 & $10.2 \%$ & $2.95[0.12,71.86]$ & & $\because$ & \\
\hline Herbst 2016 (16) & 6 & 682 & 0 & 309 & $13.9 \%$ & $5.90[0.33,104.41]$ & & & \\
\hline Mok 2019 (18) & 7 & 636 & 2 & 615 & $41.2 \%$ & $3.38[0.71,16.23]$ & & & \\
\hline Reck 2016 (21) & 3 & 154 & 0 & 150 & $10.3 \%$ & $6.82[0.36,130.90]$ & & & \\
\hline Wu 2019 (23) & 1 & 337 & 0 & 156 & $13.8 \%$ & $1.39[0.06,34.02]$ & & & \\
\hline Total $(95 \% \mathrm{Cl})$ & & 2227 & & 1627 & $100.0 \%$ & $3.90[1.41,10.81]$ & & & \\
\hline Total events & 20 & & 2 & & & & & & \\
\hline $\begin{array}{l}\text { Heterogeneity: } \mathrm{Chi}^{2}= \\
\text { Test for overall effect }\end{array}$ & $\begin{array}{l}.69, \mathrm{df}=5 \\
=2.62(\mathrm{P}\end{array}$ & $\begin{array}{l}0.98) ; \\
009)\end{array}$ & ${ }^{2}=0 \%$ & & & & $0.01 \quad 0.1$ & 1 & 10 \\
\hline
\end{tabular}

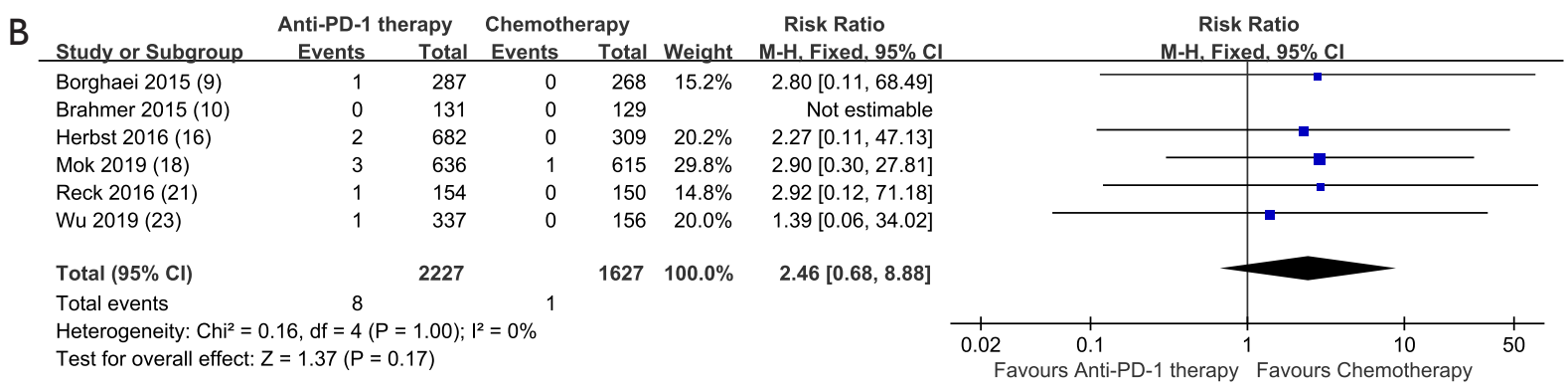

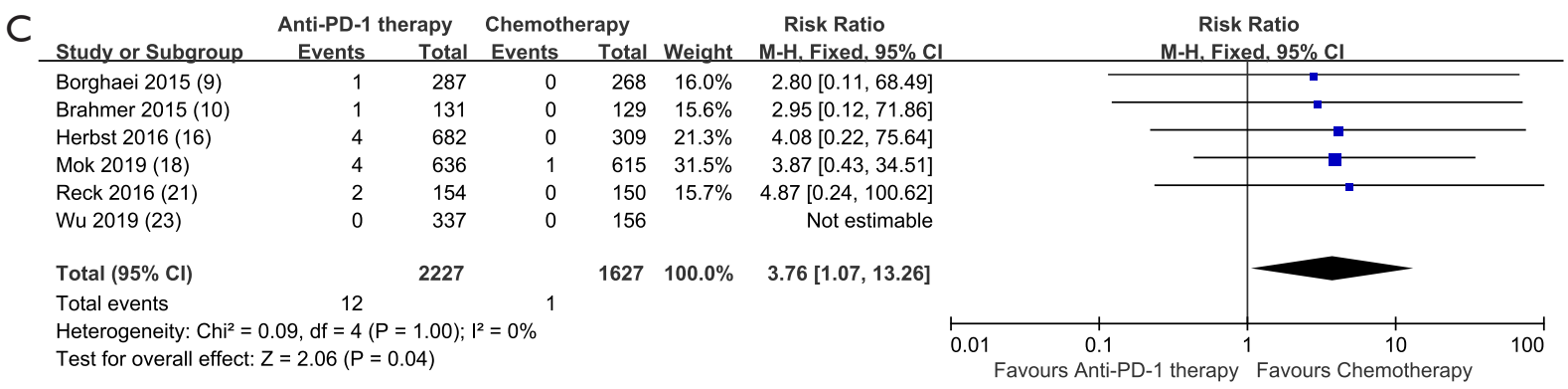

Figure 2 Forest plots for relative risk of all-grade colitis (A), grade 1-2 colitis (B) and grade 3-4 colitis (C) in advanced NSCLC patients treated with anti-PD-1 monotherapy compared with chemotherapy. NSCLC, non-small cell lung cancer.

Table 11 Sensitivity analysis for the incidence of colitis (grade 1-4) in treated with anti PD-1 monotherapy versus chemotherapy

\begin{tabular}{|c|c|c|c|c|c|}
\hline Removed study & Studies (n) & \multicolumn{2}{|c|}{ Heterogeneity } & $\mathrm{RR}(95 \% \mathrm{Cl})$ & $\mathrm{P}$ \\
\hline All study & 6 & 0.98 & 0 & $3.9(1.41-10.81)$ & 0.009 \\
\hline Borghaei (9) & 5 & 0.96 & 0 & $3.81(1.29-11.25)$ & 0.020 \\
\hline Brahmer (10) & 5 & 0.96 & 0 & $4.01(1.37-11.75)$ & 0.010 \\
\hline Mok (18) & 5 & 0.95 & 0 & $4.27(1.12-16.25)$ & 0.030 \\
\hline Reck (21) & 5 & 0.97 & 0 & 3.57 (1.2-10.63) & 0.020 \\
\hline Wu (23) & 5 & 0.99 & 0 & $4.31(1.45-12.77)$ & 0.008 \\
\hline
\end{tabular}


Table 12 Sensitivity analysis for the incidence of colitis (grade 3-4) in treated with anti PD-1 monotherapy versus chemotherapy

\begin{tabular}{|c|c|c|c|c|c|}
\hline \multirow{2}{*}{ Removed study } & \multirow{2}{*}{ Studies (n) } & \multicolumn{2}{|c|}{ Heterogeneity } & \multirow{2}{*}{$\mathrm{RR}(95 \% \mathrm{Cl})$} & \multirow{2}{*}{$\mathrm{P}$} \\
\hline & & $\mathrm{P}$ & $\mathrm{I}^{2}(\%)$ & & \\
\hline All study & 6 & 1.0 & 0 & $3.76(1.07-13.26)$ & 0.04 \\
\hline Borghaei (9) & 5 & 1.0 & 0 & $3.94(1.00-15.55)$ & 0.05 \\
\hline Brahmer (10) & 5 & 1.0 & 0 & $3.91(0.99-15.40)$ & 0.05 \\
\hline Mok (18) & 5 & 0.99 & 0 & $3.71(0.79-17.32)$ & 0.10 \\
\hline Reck (21) & 5 & 1.0 & 0 & $3.55(0.89-14.25)$ & 0.07 \\
\hline Wu (23) & 5 & 1.0 & 0 & $3.76(1.07-13.26)$ & 0.04 \\
\hline
\end{tabular}

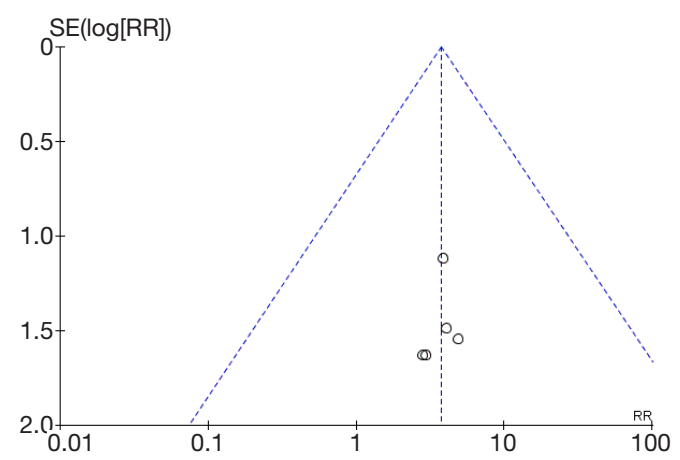

Figure 3 Funnel plots for grade $1-4$ colitis in patients treated with PD-1 inhibitors versus chemotherapy.

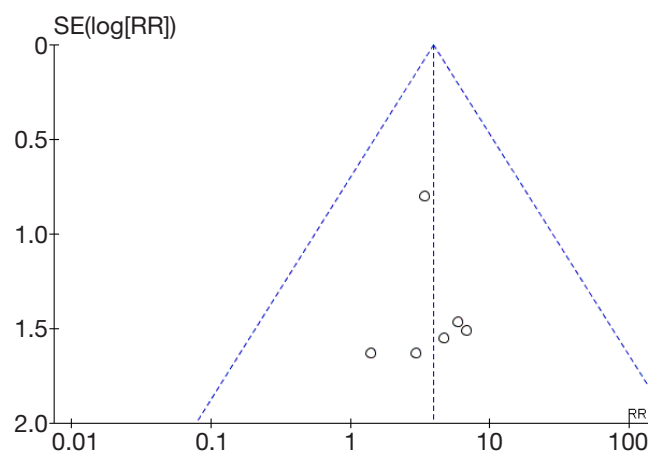

Figure 4 Funnel plots for grade 3-4 colitis in patients treated with PD-1 inhibitors versus chemotherapy.

\section{Subgroup analysis of the incidence of colitis between PD-1 inhibitors and chemotherapy}

To further investigate the effect of the specific type of PD-1 inhibitors on the RR of colitis, we performed subgroup analyses based on either nivolumab or pembrolizumab. The
RR of colitis did not differ significantly between nivolumab and chemotherapy. In contrast, pembrolizumab showed a significant increase in grade $1-4$ colitis, $\mathrm{RR}=4.46(95 \%$ CI: $1.28-15.58, \mathrm{P}=0.02)$, compared with chemotherapy $(16,18,21)$ (Figure 5).

\section{Discussion}

To the best of our knowledge, the current meta-analysis involves the largest number of patients in assessing the rates of PD-1 inhibitor-related GI adverse events in advanced NSCLC.

Based on promising results of several landmark clinical trials, the US FDA approved nivolumab and pembrolizumab for the treatment of advanced NSCLC. Pembrolizumab has also been approved for patients who are naïve to chemotherapy when the expression of $\mathrm{PD}$ L1 is greater than $50 \%(21,22)$. Besides appreciating the beneficial anti-cancer effects of PD-1 inhibitors, it is important to pay close attention to the occurrence of immune-related side reactions due to the use of these therapeutic interventions. Common drug-related adverse reactions involve the GI system and, when severe, can lead to treatment discontinuation.

Our study indicated that the most frequent GI adverse events among patients treated with PD-1 inhibitors are decreased appetite (10.0\%, 95\% CI: 8.3-11.7\%), nausea (9.2\%, 95\% CI: $7.3-11.0 \%)$ and diarrhea (8.6\%, $95 \%$ CI: 6.6-10.6\%). Other manifestations of GI adverse effects, albeit occurring less frequently, include vomiting (3.2\%, 95\% CI: $1.9-4.5 \%)$, constipation $(2.8 \%, 95 \%$ CI: $1.8-3.9 \%)$, stomatitis (1.8\%, $95 \%$ CI: $1.0-2.7 \%)$ and colitis $(0.7 \%, 95 \%$ CI: $0.4-1.1 \%)$. Of note, no patient 


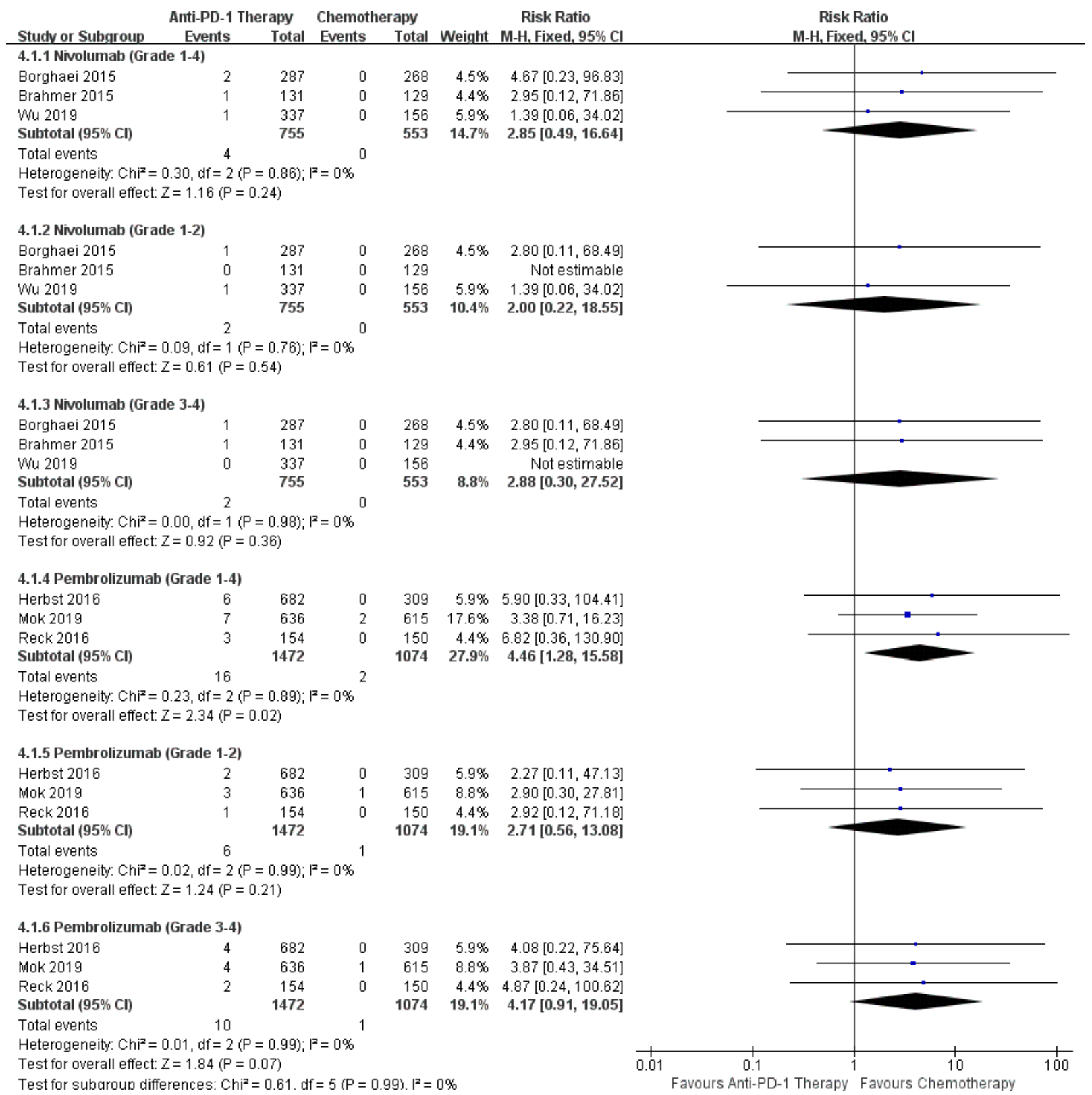

Figure 5 Forest plots analysis for colitis with different type of PD-1 inhibitors (nivolumab or pembrolizumab) monotherapy versus chemotherapy.

discontinued therapy due to decreased appetite. When compared to chemotherapy, the use of a PD-1 inhibitor carried a higher risk of all-grade colitis $(\mathrm{RR}=3.90,95 \% \mathrm{CI}$ : $1.41-10.81, \mathrm{P}=0.009)$ as well as grade $3-4$ colitis $(\mathrm{RR}=3.76$, 95\% CI: $1.07-13.26, \mathrm{P}=0.04)$. Treatment discontinuation was also frequently experienced among patients with colitis (45.7\%, 95\% CI: 20.6-70.7\%). Given that the incidence of colitis seems higher with the use of PD-1 inhibitors as opposed to the use of chemotherapy, and that nearly half of the patients discontinued therapy due to this unwanted effect, measures should be taken to monitor patients at risk of developing the condition to maximize clinical benefits of PD-1 inhibitor therapy.

The estimated incidences of GI adverse events as 
observed in our study are consistent with the results published in the report by Li et al. (24), who cite an incidence of the various GI symptoms as follows: all-grade loss of appetite $(10.8 \%)$, nausea $(9.2 \%)$, diarrhea $(8.2 \%)$, constipation $(3.9 \%)$ and vomiting $(3.7 \%)$. In their report, Wang et al. (25) gave the incidence of grade 1-4 and grade $3-4$ colitis as $0.8 \%$ and $1.2 \%$, respectively. Of note, the current meta-analysis is more comprehensive and comprises more patients compared to any of the previous studies.

In a meta-analysis by Almutairi and colleagues, nivolumab showed a significantly lower risk of nausea than pembrolizumab in patients with advanced NSCLC (26). This contradicts the results of our current study in which nivolumab had both a higher incidence of grade 1-4 and grade 3-4 nausea and colitis as compared to pembrolizumab. The discrepancy in the results of these two studies could be due to the sparse literature, only three, included in the study by Almutairi and co-workers who also conducted an indirect comparison between nivolumab and pembrolizumab.

Most manifestations of GI adverse events are easy to detect and can be identified early on. Implementing early diagnosis and prompt intervention is critical so as to optimize therapy and avoid treatment discontinuation. When adverse events occur, judicious assessments whether to suspend or to continue therapy, depending on the severity of the adverse event, should be made. When the severity of the adverse events is adequately mitigated and is no longer a threat, it is possible to consider re-introducing the use of immune checkpoint inhibitors except in special circumstances (27). Should the symptoms persist or worsen, steroids can be administered to counter the inflammation. Other treatment options include the use of mycophenolate or infliximab. Moreover, collaborative diagnosis and treatment by interdisciplinary teams that include gastroenterologists may reduce symptoms associated with these adverse events.

Although providing useful insights, we acknowledge that our study has some limitations. First, the studies that were included in the meta-analysis were conducted in an openlabel manner without the use of a placebo control arm. These studies, therefore, are prone to bias. Second, almost all the patients in the studies considered in the meta-analysis were in good physical and health condition, with relatively high tolerance to toxicity. Clinically, it is anticipated that patients are likely to have other confounding medical conditions that may further complicate the occurrence and severity of GI adverse events. Therefore, real-world evidence is necessary, to complement the trial evidence, under conditions of greater heterogeneity in patients and treatment settings. Finally, we are unable to evaluate the relationship between the occurrence of adverse events and the duration of use of PD-1 inhibitors; this requires detailed data analysis and follow-up of individual patients.

In summary, our meta-analysis recruited 15 studies and has demonstrated the GI adverse events of PD-1 monotherapy in patients with advanced/metastatic NSCLC. Colitis often resulted in therapy discontinuation. The use of PD-1 inhibitors was associated with a higher incidence of colitis as compared to chemotherapy. These findings call for caution and provide insights that should guide clinical management of patients with PD-1 inhibitors.

\section{Acknowledgments}

We would like to thank Chunxiao Yan for his help in polishing our paper.

Funding: This work was supported by funding from the Tianjin Major Disease Prevention and Control Science and Technology project (18ZXDBSY00050).

\section{Footnote}

Reporting Checklist: The authors have completed the PRISMA reporting checklist. Available at https://dx.doi. org/10.21037/tcr-21-125

Conflicts of Interest: All authors have completed the ICMJE uniform disclosure form (available at https://dx.doi. org/10.21037/tcr-21-125). The authors have no conflicts of interest to declare.

Ethical Statement: The authors are accountable for all aspects of the work in ensuring that questions related to the accuracy or integrity of any part of the work are appropriately investigated and resolved.

Open Access Statement: This is an Open Access article distributed in accordance with the Creative Commons Attribution-NonCommercial-NoDerivs 4.0 International License (CC BY-NC-ND 4.0), which permits the noncommercial replication and distribution of the article with the strict proviso that no changes or edits are made and the original work is properly cited (including links to both the formal publication through the relevant DOI and the license). See: https://creativecommons.org/ 
licenses/by-nc-nd/4.0/.

\section{References}

1. Bray F, Ferlay J, Soerjomataram I, et al. Global cancer statistics 2018: GLOBOCAN estimates of incidence and mortality worldwide for 36 cancers in 185 countries. CA Cancer J Clin 2018;68:394-424.

2. Belani CP, Marts S, Schiller J, et al. Women and lung cancer: epidemiology, tumor biology, and emerging trends in clinical research. Lung Cancer 2007;55:15-23.

3. Jia L, Zhang Q, Zhang R. PD-1/PD-L1 pathway blockade works as an effective and practical therapy for cancer immunotherapy. Cancer Biol Med 2018;15:116-23.

4. Gupta A, De Felice KM, Loftus EV Jr, et al. Systematic review: colitis associated with anti-CTLA-4 therapy. Aliment Pharmacol Ther 2015;42:406-17.

5. Slim K, Nini E, Forestier D, et al. Methodological index for non-randomized studies (minors): development and validation of a new instrument. ANZ J Surg 2003;73:712-6.

6. Basch E, Reeve BB, Mitchell SA, et al. Development of the National Cancer Institute's patient-reported outcomes version of the common terminology criteria for adverse events (PRO-CTCAE). J Natl Cancer Inst 2014;106:dju244.

7. DerSimonian R, Laird N. Meta-analysis in clinical trials revisited. Contemp Clin Trials 2015;45:139-45.

8. Higgins JP, Thompson SG, Deeks JJ, et al. Measuring inconsistency in meta-analyses. BMJ 2003;327:557-60.

9. Borghaei H, Paz-Ares L, Horn L, et al. Nivolumab versus Docetaxel in Advanced Nonsquamous Non-Small-Cell Lung Cancer. N Engl J Med 2015;373:1627-39.

10. Brahmer J, Reckamp KL, Baas $\mathrm{P}$, et al. Nivolumab versus Docetaxel in Advanced Squamous-Cell Non-Small-Cell Lung Cancer. N Engl J Med 2015;373:123-35.

11. Carbone DP, Reck M, Paz-Ares L, et al. First-Line Nivolumab in Stage IV or Recurrent Non-Small-Cell Lung Cancer. N Engl J Med 2017;376:2415.

12. Garon EB, Rizvi NA, Hui R, et al. Pembrolizumab for the treatment of non-small-cell lung cancer. N Engl J Med 2015;372:2018-28.

13. Gettinger S, Rizvi NA, Chow LQ, et al. Nivolumab Monotherapy for First-Line Treatment of Advanced NonSmall-Cell Lung Cancer. J Clin Oncol 2016;34:2980-7.

14. Goldberg SB, Gettinger SN, Mahajan A, et al. Pembrolizumab for patients with melanoma or non-smallcell lung cancer and untreated brain metastases: early analysis of a non-randomised, open-label, phase 2 trial.
Lancet Oncol 2016;17:976-83.

15. Hellmann MD, Rizvi NA, Goldman JW, et al. Nivolumab plus ipilimumab as first-line treatment for advanced nonsmall-cell lung cancer (CheckMate 012): results of an open-label, phase 1, multicohort study. Lancet Oncol 2017;18:31-41.

16. Herbst RS, Baas P, Kim DW, et al. Pembrolizumab versus docetaxel for previously treated, PD-L1-positive, advanced non-small-cell lung cancer (KEYNOTE-010): a randomised controlled trial. Lancet 2016;387:1540-50.

17. Hida T, Nishio M, Nogami N, et al. Efficacy and safety of nivolumab in Japanese patients with advanced or recurrent squamous non-small cell lung cancer. Cancer Sci 2017;108:1000.

18. Mok TSK, Wu YL, Kudaba I, et al. Pembrolizumab versus chemotherapy for previously untreated, PD-L1expressing, locally advanced or metastatic non-small-cell lung cancer (KEYNOTE-042): a randomised, open-label, controlled, phase 3 trial. Lancet 2019;393:1819-30.

19. Nishio M, Hida T, Atagi S, et al. Multicentre phase II study of nivolumab in Japanese patients with advanced or recurrent non-squamous non-small cell lung cancer. ESMO Open 2017;1:e000108.

20. Nishio M, Takahashi T, Yoshioka H, et al. KEYNOTE-025: Phase 1b study of pembrolizumab in Japanese patients with previously treated programmed death ligand 1-positive advanced non-small-cell lung cancer. Cancer Sci 2019;110:1012-20.

21. Reck M, Rodríguez-Abreu D, Robinson AG, et al. Pembrolizumab versus Chemotherapy for PD-L1Positive Non-Small-Cell Lung Cancer. N Engl J Med 2016;375:1823-33.

22. Rizvi NA, Mazières J, Planchard D, et al. Activity and safety of nivolumab, an anti-PD-1 immune checkpoint inhibitor, for patients with advanced, refractory squamous non-small-cell lung cancer (CheckMate 063): a phase 2, single-arm trial. Lancet Oncol 2015;16:257.

23. Wu YL, Lu S, Cheng Y, et al. Nivolumab Versus Docetaxel in a Predominantly Chinese Patient Population With Previously Treated Advanced NSCLC: CheckMate 078 Randomized Phase III Clinical Trial. J Thorac Oncol 2019;14:867-75.

24. Li ZQ, Yan HC, Gu JJ, et al. Comparative efficacy and safety of PD-1/PD-L1 Inhibitors versus platinum-based chemotherapy for the first-line treatment of advanced non-small cell lung cancer: a meta analysis of randomized controlled trials. Pharmacol Res 2020;160:105194.

25. Wang DY, Ye F, Zhao S, et al. Incidence of immune 
checkpoint inhibitor-related colitis in solid tumor patients: A systematic review and meta-analysis. Oncoimmunology 2017;6:e1344805.

26. Almutairi AR, Alkhatib N, Martin J, et al. Comparative efficacy and safety of immunotherapies targeting the PD-1/PD-L1 pathway for previously treated advanced non-small cell lung cancer: A Bayesian network meta-

Cite this article as: Huo GW, Zhu FY, Zuo R, Song Y, Chen WD, Chen WM, Zhang HM, Jia SS, Chen P. The incidence of gastrointestinal adverse events in patients with advanced non-small cell lung cancer (NSCLC) treated with PD-1 inhibitors: a meta-analysis. Transl Cancer Res 2021;10(7):3389-3403. doi: 10.21037/tcr-21-125 analysis. Crit Rev Oncol Hematol 2019;142:16-25.

27. Brahmer JR, Lacchetti C, Schneider BJ, et al. Management of Immune-Related Adverse Events in Patients Treated With Immune Checkpoint Inhibitor Therapy: American Society of Clinical Oncology Clinical Practice Guideline. J Clin Oncol 2018;36:1714-68. 